\title{
A LINKAGE OF MIND AND BRAIN: SIR JOHN ECCLES AND MODERN DUALISTIC INTERACTIONISM
}

\section{Danko Georgiev}

Department of Psychiatry and Neurobiology, Graduate School of Medical Science, Kanazawa University, Kanazawa, Japan

Our minds, constituted by conscious experiences, are both the most familiar and most mysterious aspect of our lives. Despite the large amount of clinical evidence suggesting an intimate relationship between the brain function and the mind, the nature of this relationship remains poorly understood. In this Commentary we discuss some of the problems faced by the classical mind-brain identity theory and explain how the quantum dualistic interactionism proposed by Sir John Eccles could resolve these problems.

Biomed Rev 2011; 22: 81-84.

Key words: mind, conscious experience, brain, materialism, dualism, quantum mechanics

\section{MIND-BRAIN PROBLEM IN CLASSICAL PHYSICS}

Our minds are constituted by subjective conscious experiences through which we access ourselves and the surrounding world. Examples of conscious experiences are the pain of the toothache, the smell of the rose, or the perceived blueness of the blue sky. Large amount of clinical evidence suggests that there is an intimate relationship between the brain function and the mind, because discrete lesions in the brain could impair our cognitive abilities and change the way we experience the world (1). Thus it is clear that our minds should depend somehow on the brain states. Nevertheless, this is the best that classical materialism can say on the subject. Asking further questions within the framework of classical materialism leads to paradoxical conclusions, which contradict experimental observations and our common sense. Several important questions are:

(i) How the mind can affect the physical brain?

(ii) How can we have free will if we cannot choose between several alternatives?

(iii) How can we be responsible for our actions if our choices are predetermined?

(iv) Why the brain processes produce any experiences at all?

Received 20 December 2011, accepted 22 December 2011.

Correspondence: Danko Georgiev MD, PhD, Department of Psychiatry and Neurobiology, Graduate School of Medical

Science, Kanazawa University, 13-1 Takaramachi, Kanazawa, Ishikawa 920-8641, Japan.

E-mail: danko@med.kanazawa-u.ac.jp 
(v) Why during anesthesia the brain processes do not produce experiences?

According to classical materialism all brain processes can be reduced to molecular chains of causes and effects, which although immensely complex operate as a deterministic clockwork mechanism (2). Because the material world is causally closed, if the brain states produce conscious experiences, then these experiences cannot be causally effective. According to the evolution theory however, something that is not causally effective cannot lead to evolutionary advantage and cannot be selected by natural selection. The mind-brain identity theory is an attempt to resolve this problem. It postulates that the mind states are brain states, which makes the mind states causally effective and capable of providing evolutionary advantage. The proposed solution however comes at a dear price. Because the mind-brain identity theory is based on classical materialism, the brain dynamics is completely deterministic. Therefore, the mind-brain choices are predetermined and there is no room for free will or choice making. If we cannot make choices we cannot be morally responsible for our actions, no more than a falling stone is morally responsible for breaking one's leg. Furthermore, by attributing mental properties to the material brain states, it is not easy to explain why there are brain processes that do not produce conscious experiences. For example, during general anesthesia, the conscious experience is safely erased, yet, an experimentalist can flash light into the eye of an anesthetized animal and still record evoked potentials from pyramidal neurons in the primary visual cortex (3). If mind states are identical to brain states, it should be impossible to turn them on or off using anesthetics, because the brain states always remain brain states.

In a Dance Round in this volume of Biomedical Reviews, it was suggested that the mind-brain identity thesis applies to "events of sensations" and "distributed processes in the brain" (4). Such a definition however is vacuous. There are various distributed processes in the brain that do not produce conscious experiences. Furthermore, the events of sensations are by definition mental and to say that conscious experiences are associated with brain states or processes that produce sensation is circular. One might be tempted to identify the events of sensations with the electrophysiological processes of inputting sensory information from the sense organs to the cortex, but certainly the latter processes are not always associated with sensory experiences as can be seen from the provided example with recording of visually evoked potentials from pyramidal neurons in the primary sensory cortex of anesthetized animals (3). Experiments with similar results were performed also in anesthetized human patients using auditory stimulation and EEG recording (5).

\section{MIND-BRAIN PROBLEM IN QUANTUM PHYSICS}

The ease with which one can construct arguments contradicting experimental observations and our common sense does not solely result from the postulated mind-brain identity. Instead, the roots of the problem lie within the causally closed deterministic structure of classical materialism. Fortunately, in 1920's with the birth of quantum mechanics, which describes the behavior of elementary physical particles, it became clear that classical materialism is not a correct description of the physical world. The behavior of elementary physical particles was found to be inherently indeterministic so that one cannot predict exactly the future state of an individual particle, only the probability with which given future state could occur. The dynamics of individual quantum particles is governed by a wavefunction $\psi$, which is obtained by solving the Schrödinger equation (6). The wavefunction $\psi$ depends on the boundary conditions that include the initial position of the particle and its environment. If one knows the boundary conditions, one can solve the Schrödinger equation and see how the wavefunction $\psi$ evolves in a deterministic way through space and time. What makes the theory extraordinary is the fact that the wavefunction $\psi$ of a quantum system cannot be observed. The wavefunction $\psi$ is sometimes described as pre-probability because it is the square of the wavefunction $|\psi|^{2}$ that gives the probability to find the quantum particle at a certain position at a certain time (7). Curiously, the wavefunction $\psi$ is defined in a non-material multi-dimensional Hilbert space, in which each axis represents a possible state of the quantum particle. If the quantum particle can be in infinite number of possible states, the Hilbert space is infinite-dimensional $(8,9)$. The indeterminism in quantum mechanics stems from the fact that when the particle interacts with other particles only one of the possible states is actualized. After the interaction the new position of the particle and its new environment will serve as new boundary conditions for solving the Schrödinger equation and obtaining new wavefunction $\psi$. The new wavefunction $\psi$ will evolve again in a deterministic way through space and time until the next interaction, during which one of the multiple possibilities will be actualized with the square of the new wavefunction $|\psi|^{2}$ providing the probability to find the quantum particle at a certain position at a certain time. Undoubtedly such behavior of the elementary physical particles may look disturbing and incomprehensible at first encounter. Richard Feynman, who won the 1965 Nobel Prize in Physics for his work on quantum electrodynamics, repeatedly stressed that nobody understands how the real world can be like this (10). And when asked whether he likes the weird quantum laws, he answered that it is irrelevant what he likes or dislikes, the important thing is how the physical world is. The predictions of quantum theory are currently confirmed with such a high degree of precision 
that there is little doubt that quantum theory is the correct physical description of the world. Sir John Eccles, who won the 1963 Nobel Prize in Physiology or Medicine for his work on the synapse, was one of the first who understood the importance of quantum mechanics for resolving the mind-brain problem, and proposed that mental events can cause brain events analogously to how the wavefunction $\psi$ determines the probability $|\psi|^{2}$ for a given quantum particle to be found at a certain position at a certain time (11). Indeed if one considers the wavefunction $\psi$ of a quantum particle as a non-observable mental state of pre-probabilities and the actualized position in space and time as an observable material state, then the dualistic interactionism proposed by Sir John Eccles is consistent with the modern vision of what the physical world is. Interestingly, in quantum mechanics a system of interacting quantum particles can have a wavefunction $\psi$ satisfying the Schrödinger equation, whereas the individual particles in the composite system may not have individual wavefunctions $\psi$. Such state does not have a classical analogue, and is referred to as a quantum entangled state (9). If the human mind could be described by a global wavefunction $\psi$ that results from quantum entanglement of different brain subcomponents, each of which does not have an individual wavefunction $\psi$, then one sees that it would be incorrect to say that the mind represented by the global wavefunction $\psi$ is composed of simpler minds. Furthermore, in dualistic interactionism general anesthesia and loss of consciousness should not be understood simply as turning off the mental states. Instead following the analogy with split-brain patients, who have two independent minds in each cerebral hemisphere due to surgically severed corpus callosum (12), one can assume that anesthetic molecules disrupt the interactions and the quantum entanglements between individual brain molecules leading to zillion split-brain subcomponents, each of which possessing the simplest mental states available to individual quantum particles. Thus consciousness would not emerge out of nothing and would not disintegrate down to nothing. In its essence the dualism affirms that consciousness can have different level of complexity with simplest mental properties attributable to all elementary quantum particles.

The conceptual difference between the dualistic interactionism based on quantum mechanics, and alternative versions of dualism based on classical materialism, such as epiphenomenalism or psychophysic parallelism, is shown in Figure 1. Because quantum laws are indeterministic it is possible to construct a theory in which the mind and brain states interact: the brain state produces deterministically a mind state after which the mind state makes an indeterministic choice from multiple possible alternatives and selects a future brain state. This is in stark contrast with classical materialism where the brain states obey causally closed deterministic laws. In epiphenomenalism, the brain states produce the mental states but these mental states are unable to affect causally the brain states. The epiphenomenal mental states are just useless spectators. Even more ridiculous is the psychophysical parallelism where the brain states and the mental states do not interact at all, but which are set in a pre-established harmony created by a divine creator.

\section{Dualist interactionism}

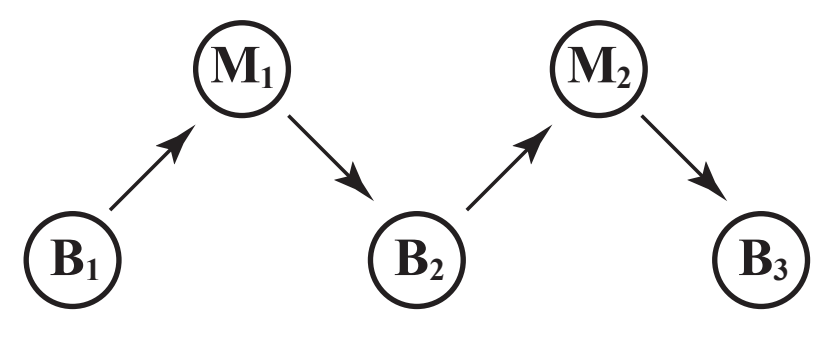

Epiphenomenalism

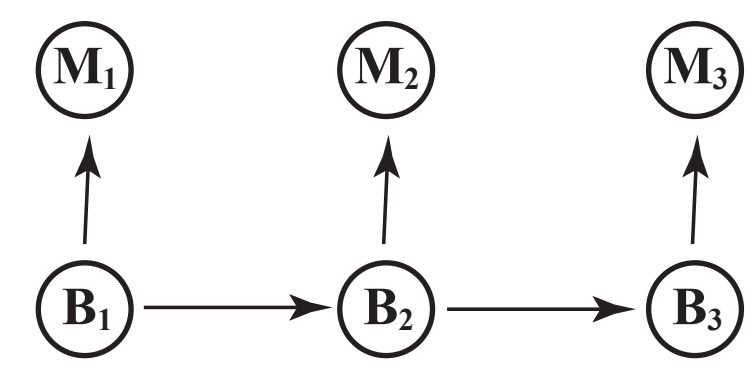

Psychophysic parallelism

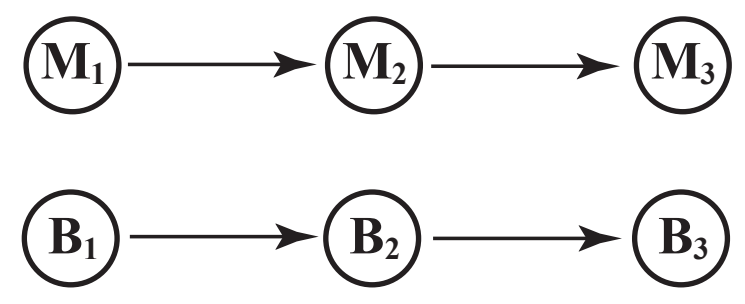

Figure 1. Varieties of dualist causal interaction. Dualist interactionism affirms that brain states produce mental states, which may in turn select the future brain states. Epiphenomenalism affirms that brain states produce mental states, which do not affect causally the brain states. Psychophysic parallelism affirms that brain states and mental states do not interact with each other but are set in a pre-established harmony created by a divine creator. $B_{1}, B_{2}, B_{3}$, brain states; $M_{1}, M_{2}, M_{3}$, mental states; arrows indicate the direction of the interactions. 


\section{CONCLUSIONS}

In a Dance Round in this $B M R$ volume, it was stated that dualism is "undeniably suspended with the data of neuroscience" and that "besides Sir John Eccles more than forty years ago, there is no other scientist from the field of empirical science to any more hold the position of dualism" (4). While any form of dualism based on classical materialism can be considered refuted, it is not true that dualism in general is refuted and that no present scientist holds such position. Currently, an increasing number of neuroscientists and physicists think that quantum theory could support dualist interactionism along the lines envisaged by Sir John Eccles. Attestation for that are the plethora of recent models based on the nanoscale organization of the neurons in which the mind states can affect the brain through quantum effects (13-19). Some support for the feasibility of such quantum models has been already provided by the experimental verification of quantum tunneling for hydrogen transfer in enzyme-catalyzed reactions (20-22), quantum quasi-particle assisted folding of proteins (23) and microsecond quantum coherence in the retina of some birds that are able to navigate by sensing Earth's magnetic field (24). The dualist interactionism envisaged by Sir John Eccles is not only viable, it could be our best bet for a physical theory of consciousness.

\section{REFERENCES}

1. Gross CG. A Hole in the Head: More Tales in the History of Neuroscience. MIT Press, 2009.

2. James W. Are we automata? Mind 1879; 4: 1-22.

3. Yu S, Yang H, Nakahara H, Santos GS, Nikolić D, Plenz D. Higher-order interactions characterized in cortical activity. J Neurosci 2011; 31: 17514-17526.

4. Stoyanov D. A linkage of mind and brain: towards translational validity between neurobiology and psychiatry. Biomed Rev 2011; 22: 65-76.

5. McNeer RR, Bohórquez J, Özdamar Ö. Influence of auditory stimulation rates on evoked potentials during general anesthesia: relation between the transient auditory middle-latency response and the $40-\mathrm{Hz}$ auditory steady state response. Anesthesiology 2009; 110: 1026-1035.

6. Schrödinger E. An undulatory theory of the mechanics of atoms and molecules. Phys Rev 1926; 28: 1049-1070.

7. Born M. Zur Quantenmechanik der Stoßvorgänge. Zeitschrift für Physik A 1926; 37: 863-867.
8. Susskind L. Quantum Mechanics. Stanford Continuing Studies course, Stanford University, 2008.

9. Susskind L. Quantum Entanglements. Stanford Continuing Studies course, Stanford University, 2006.

10. Feynman RP. The Douglas Robb Memorial Lectures. University of Auckland, New Zealand, 1979.

11. Eccles JC. Do mental events cause neural events analogously to the probability fields of quantum mechanics? Proc R Soc Lond B Biol Sci 1986; 227: 411-428.

12. Gazzaniga MS. The split brain revisited. Sci Am 2002; 12: 26-31.

13. Beck F, Eccles JC. Quantum aspects of brain activity and the role of consciousness. Proc Natl Acad Sci USA 1992; 89: 11357-11361.

14. Beck F. Can quantum processes control synaptic emission? Int J Neural Syst 1996; 7: 343-353.

15. Beck F, Eccles JC. Quantum processes in the brain: A scientific basis of consciousness. Cogn Stud 1998; 5: 95-109.

16. Beck F. Synaptic quantum tunneling in brain activity. NeuroQuantology 2008; 6: 140-151.

17. Penrose R. Shadows of the Mind: A Search for the Missing Science of Consciousness. Oxford University Press, 1994

18. Schwartz JM, Stapp HP, Beauregard M. Quantum physics in neuroscience and psychology: a neurophysical model of mind-brain interaction. Philos Trans R Soc Lond B Biol Sci 2005; 360: 1309-1327.

19. Stapp HP. Quantum interactive dualism: an alternative to materialism. J Consc Stud 2005; 12: 43-58.

20. Basran J, Sutcliffe MJ, Scrutton NS. Enzymatic H-transfer requires vibration driven extreme tunneling. Biochemistry 1999; 38: 3218-3222.

21. Knapp MJ, Klinman JP. Environmentally coupled hydrogen tunneling. Linking catalysis to dynamics. Eur $J$ Biochem 2002; 269: 3113-3121.

22. Sutcliffe MJ, Scrutton NS. Enzyme catalysis: Over-thebarrier or through-the-barrier? Trends Biochem Sci 2000; 25: 405-408.

23. Caspi S, Ben-Jacob E. Conformation changes and folding of proteins mediated by Davydov's soliton. Phys Lett A 2000; 272: 124-129.

24. Gauger E, Rieper E, Morton JJL, Benjamin SC, Vedral $\mathrm{V}$. Sustained quantum coherence and entanglement in the avian compass. Phys Rev Lett 2011; 106: 040503. 